\title{
Tangency quantum cohomology
}

\author{
Joachim Kock
}

\begin{abstract}
Let $X$ be a smooth projective variety. Using modified psi classes on the stack of genus-zero stable maps to $X$, a new associative quantum product is constructed on the cohomology space of $X$. When $X$ is a homogeneous variety, this structure encodes the characteristic numbers of rational curves in $X$, and specialises to the usual quantum product upon resetting the parameters corresponding to the modified psi classes. For $X=\mathbb{P}^{2}$, the product is equivalent to that of the contact cohomology of Ernström and Kennedy.
\end{abstract}

\section{Introduction}

\section{Quantum cohomology and enumerative geometry}

Let $X$ be a smooth projective variety over the complex numbers. The Gromov-Witten invariants of $X$ are constructed by pulling back cohomology classes to stacks of stable maps to $X$ and integrating over the virtual fundamental class (cf. Behrend and Manin [BM96], Behrend [Beh97], Li-Tian [LT98]). Let $\Phi$ denote the generating function for the genus-zero Gromov-Witten invariants. The quantum product is defined on the cohomology space of $X$ by taking the third derivatives of $\Phi$ as structure constants

$$
T_{i} * T_{j}:=\sum_{e, f} \Phi_{i j e} g^{e f} T_{f}
$$

cf. Kontsevich and Manin [KM94]. The associativity of this product is equivalent to the fact that $\Phi$ satisfies the WDVV equations

$$
\sum_{e, f} \Phi_{i j e} g^{e f} \Phi_{f k l}= \pm \sum_{e, f} \Phi_{j k e} g^{e f} \Phi_{f i l} .
$$

The WDVV equations first appeared as an integrability condition in topological field theory (cf. Witten [Wit91]). Their axiomatic and coordinate free treatment led to the notion of Frobenius manifolds (due to Dubrovin [Dub96]; see also Manin [Man99]), other examples of which include spaces of isomonodromic deformations and unfolding spaces of isolated hypersurface singularities.

A key feature of quantum cohomology is its connection to enumerative geometry: when $X$ is a homogeneous variety, the Gromov-Witten invariants count rational curves in $X$ subject to incidence conditions, and then the WDVV equations provide relations among the numbers, in many cases enough to determine all the numbers from a few initial ones. In this way, enumerative problems which have resisted more than a century of active research find their solution as an instance of associativity, arguably one of the most basic concepts of mathematics.

Received 17 December 2001, accepted in final form 2 January 2003.

2000 Mathematics Subject Classification 14N35 (primary), 53D45 (secondary).

Keywords: quantum cohomology, Gromov-Witten invariants, enumerative geometry.

The author was supported by the National Science Research Council of Denmark.

This journal is (c) Foundation Compositio Mathematica 2004. 


\section{J. KOCK}

\section{Gravitational quantum cohomology}

An important generalisation of Gromov-Witten invariants is the notion of descendant GromovWitten invariants (gravitational descendants), defined by incorporating psi classes (cotangent line classes) in the integrals. This corresponds in physics to coupling the topological field theory to gravity (cf. Witten [Wit91]). The quantum product easily generalises to this setting, now taking $\Phi$ to be the full gravitational Gromov-Witten potential. However, this 'gravitational' quantum product has not brought about as much interest as the first one, for several reasons. First of all, the gravitational descendants are not directly interpretable in enumerative geometry. Another reason is that the fundamental class of $X$ is no longer 1-element for the multiplication, and the structure does not fit so well into the Frobenius manifold framework.

\section{Characteristic numbers}

In enumerative geometry, one of the central problems since the time of Chasles, Zeuthen and Schubert is the characteristic number problem. The characteristic numbers are the numbers of curves subject to incidence conditions and tangency conditions. From a modern viewpoint the main interest is not so much the numbers themselves but rather the structures that govern them, so naturally the central object of study is their generating function, the characteristic number potential. While a closed formula for the characteristic number potential seems out of reach, the goal is to constrain it with differential equations. The techniques of stable maps have engendered various approaches to this problem, and a couple of good differential equations have been found, sufficient to determine the characteristic numbers of rational curves in a homogeneous variety (see Graber, Kock, and Pandharipande [GKP02] and the references given therein). However, these approaches are not related to the associativity formalism. The exception is Ernström and Kennedy [EK99], who constructed a 'contact cohomology ring' for $\mathbb{P}^{2}$, whose associativity amounts to a differential equation for the generating function for the characteristic numbers of rational plane curves. Their construction (and the proof of associativity) is very complicated though, and the method seems to be peculiar to $\mathbb{P}^{2}$.

\section{Tangency quantum cohomology}

The present work introduces a new generalisation of quantum cohomology (for any smooth projective $X$ ), which links the characteristic number problem to gravitational quantum cohomology, and puts new focus on the WDVV equations. The main result can be stated as the associativity of a tangency quantum product, expressed by certain deformed WDVV equations. While the whole construction relies on descendant invariants and is closely related to the 'gravitational' quantum product, its motivation lies in enumerative geometry: when $X$ is a homogeneous variety, the generating function for the characteristic numbers of rational curves in $X$ satisfies these deformed WDVV equations. In this way, surprisingly, the characteristic numbers are revealed to be governed by the same simple principle as the incidence-only numbers. (For $X=\mathbb{P}^{2}$ we recover the contact product of Ernström and Kennedy [EK99].)

Now follows a brief outline of the paper.

\section{The tangency quantum potential}

The construction is based on a new potential $\Gamma$, called the tangency quantum potential (introduced in $\S 1$ ), which incorporates one level of modified psi classes. Modified psi classes are boundary corrections of the tautological psi classes motivated from enumerative geometry: tangency conditions are easily expressible in terms of modified psi classes, cf. [GKP02]. In contrast to the tautological psi classes, the modified ones restrict to the boundary in a non-trivial way, giving rise to certain diagonal classes. Therefore, the Splitting Lemma 1, which is the engine in the proof of the associativity, is complicated by correction terms. 


\section{The deformed metric and the tangency WDVV equations}

The mess of correction terms is handled by the introduction of a new 'metric' $\gamma_{e f}$ on the cohomology space of $X$, which is the subject of $\S 2$. It is a deformation of the Poincaré metric $g_{e f}$ in much the same way as the tangency quantum potential is a deformation of the Gromov-Witten potential. Amazingly it encodes all the combinatorics of the diagonal corrections in the splitting lemma, leading to certain 'deformed' WDVV equations for the tangency potential, which are established in $\S 3$. These equations express the associativity of the tangency quantum product, defined as

$$
T_{i} * T_{j}:=T_{i} \cup T_{j}+\sum_{e, f} \Gamma_{i j e} \gamma^{e f} T_{f}
$$

The deformed metric is also essential for the formulation of the differential equations of [GKP02] and [Koc03].

\section{Frobenius structure}

The last section is devoted to a technical detail: since modified psi classes are not defined in degree zero, the same goes for the tangency potential. The missing degree-zero part is now constructed separately to account for the classical part of the new product. This construction shows that the tangency quantum product is in fact 'integrable' in the sense that its structure constants (with respect to $\gamma$ ) are the third derivatives of a single function. With this last piece of data, the results of the paper can be summarised in saying that the new structure is that of a formal Frobenius (super)manifold (over the power series ring in the new variables).

\section{First enumerative descendants}

\subsection{Set-up}

Throughout we work over the field of complex numbers. Let $X$ denote a smooth projective variety, and let $T_{0}, \ldots, T_{r}$ denote the elements of a homogeneous basis of the cohomology space $H^{*}(X, \mathbb{Q})$, with $T_{0}$ the fundamental class. As in Manin's book [Man99] we consider $H^{*}(X, \mathbb{Q})$ as a linear supermanifold.

Let $\bar{M}_{0, S}(X, \beta)$ denote the moduli stack of Kontsevich stable maps of genus zero whose direct image in $X$ is of class $\beta \in H_{2}^{+}(X, \mathbb{Z})$, and whose marking set is $S=\left\{p_{1}, \ldots, p_{n}\right\}$. For each mark $p_{i}$, let $\nu_{i}: \bar{M}_{0, S}(X, \beta) \rightarrow X$ denote the evaluation morphism that sends the class of a map $\mu$ to $\mu\left(p_{i}\right)$; pullbacks along evaluation morphisms of cohomology classes of $X$ are referred to as evaluation classes. The reader is referred to Fulton and Pandharipande [FP95] for definitions and basic properties of stable maps, Gromov-Witten invariants, and quantum cohomology.

\subsection{Modified psi classes (cf. [GKP02])}

Let $\pi_{0}: \bar{M}_{0, S \cup\left\{p_{0}\right\}}(X, \beta) \rightarrow \bar{M}_{0, S}(X, \beta)$ denote the forgetful morphism that forgets the extra mark $p_{0}$. Together with the sections $\sigma_{i}$ corresponding to each of the marks in $S$, it constitutes the universal family. For each mark there is a psi class defined as $\boldsymbol{\psi}_{i}:=c_{1}\left(\sigma_{i}^{*} \omega_{\pi_{0}}\right)$, where $\omega_{\pi_{0}}$ is the relative dualising sheaf of $\pi_{0}$.

When $\beta \neq 0$, the modified psi class is $\overline{\boldsymbol{\psi}}_{i}:=\hat{\pi}_{i}^{*} \boldsymbol{\psi}_{i}$, where $\hat{\pi}_{i}: \bar{M}_{0, S}(X, \beta) \rightarrow \bar{M}_{0,\left\{p_{i}\right\}}(X, \beta)$ is the forgetful morphism that forgets all marks but $p_{i}$.

Modified psi classes arise naturally in enumerative geometry: for example, if $X$ is a homogeneous variety and $Z \subset X$ is a very ample hypersurface of degree $\mathbf{z} \in H^{2}(X, \mathbb{Q})$, then the cycle of maps tangent to $Z$ at $p_{i}$ is of class $\nu_{i}^{*}(\mathbf{z}) \cup\left(\overline{\boldsymbol{\psi}}_{i}+\nu_{i}^{*}(\mathbf{z})\right)$. It then follows from a transversality argument that the characteristic numbers of rational curves are products of top dimension of evaluation classes and 


\section{J. KOCK}

modified psi classes (integrated over the virtual fundamental class of $\bar{M}_{0, S}(X, \beta)$ ). For this reason, these top products,

$$
\left\langle\bar{\tau}_{m_{1}}\left(\mathbf{z}_{1}\right) \cdots \bar{\tau}_{m_{n}}\left(\mathbf{z}_{n}\right)\right\rangle_{\beta}:=\int \bar{\psi}_{1}^{m_{1}} \cup \nu_{1}^{*}\left(\mathbf{z}_{1}\right) \cup \cdots \cup \bar{\psi}_{n}^{m_{n}} \cup \nu_{n}^{*}\left(\mathbf{z}_{n}\right) \cap\left[\bar{M}_{0, S}(X, \beta)\right]^{\text {virt }},
$$

are called enumerative descendants in [GKP02].

The enumerative descendants that appear in the characteristic number problem are only the first enumerative descendants, i.e. those made up of factors of type $\bar{\tau}_{0}(\mathbf{x})$ and $\bar{\tau}_{1}(\mathbf{y})$, for $\mathbf{x}, \mathbf{y} \in H^{*}(X, \mathbb{Q})$. For simplicity, we will work in the fixed basis $T_{0}, \ldots, T_{r}$ and employ the following notation for the corresponding integrals:

$$
\left\langle\overline{\boldsymbol{\tau}}_{0}^{\mathbf{a}} \overline{\boldsymbol{\tau}}_{1}^{\mathbf{b}}\right\rangle_{\beta}:=\left\langle\prod_{k=0}^{r}\left(\bar{\tau}_{0}\left(T_{k}\right)\right)^{a_{k}}\left(\bar{\tau}_{1}\left(T_{k}\right)\right)^{b_{k}}\right\rangle_{\beta},
$$

where $\mathbf{a}=\left(a_{0}, \ldots, a_{r}\right)$ and $\mathbf{b}=\left(b_{0}, \ldots, b_{r}\right)$ are vectors of non-negative integers. The integral is zero unless $\sum_{k}\left(\frac{1}{2} \operatorname{deg}\left(T_{k}\right)\left(a_{k}+b_{k}\right)+b_{k}\right)$ equals the virtual dimension of $\bar{M}_{0, S}(X, \beta)$, where $S$ is of cardinality $n=\sum\left(a_{k}+b_{k}\right)$.

\subsection{Diagonal classes (cf. [GKP02])}

For $\beta>0$, the diagonal class $\boldsymbol{\delta}_{i j} \in H^{2}\left(\bar{M}_{0, S}(X, \beta), \mathbb{Q}\right)$ is defined as the pull-back from $\bar{M}_{0,\left\{p_{i}, p_{j}\right\}}(X, \beta)$ of the Cartier divisor $D_{i j}$ (consisting of maps having both marks on a contracting twig). It can also be described as the sum of all boundary divisors having $p_{i}$ and $p_{j}$ together on a contracting twig.

Let $\pi_{0}: \bar{M}_{0, S \cup\left\{p_{0}\right\}}(X, \beta) \rightarrow \bar{M}_{0, S}(X, \beta)$ denote the morphism that forgets $p_{0}$. Then

$$
\pi_{0 *}\left(\boldsymbol{\delta}_{0 i} \cap\left[\bar{M}_{0, S \cup\left\{p_{0}\right\}}(X, \beta)\right]^{\mathrm{virt}}\right)=\left[\bar{M}_{0, S}(X, \beta)\right]^{\mathrm{virt}}
$$

in $H_{*}\left(\bar{M}_{0, S}(X, \beta), \mathbb{Q}\right)$.

The diagonal classes owe their name to the following properties:

$$
\begin{aligned}
\boldsymbol{\delta}_{i j} \boldsymbol{\delta}_{i k} & =\boldsymbol{\delta}_{i j} \boldsymbol{\delta}_{j k} \\
-\boldsymbol{\delta}_{i j}^{2}=\boldsymbol{\delta}_{i j} \overline{\boldsymbol{\psi}}_{i} & =\boldsymbol{\delta}_{i j} \overline{\boldsymbol{\psi}}_{j} \\
\boldsymbol{\delta}_{i j} \nu_{i}^{*}(\mathbf{z}) & =\boldsymbol{\delta}_{i j} \nu_{j}^{*}(\mathbf{z}) \quad \text { for } \mathbf{z} \in H^{*}(X, \mathbb{Q}) .
\end{aligned}
$$

The swapping property also holds when $\boldsymbol{\delta}_{i j}$ is replaced with $D_{i j}$.

\subsection{Key formula}

Let $D=D\left(S^{\prime}, \beta^{\prime} \mid S^{\prime \prime}, \beta^{\prime \prime}\right)$ denote the boundary divisor which is the image of the morphism

$$
\rho_{D}: \bar{M}_{0, S^{\prime} \cup\left\{x^{\prime}\right\}}\left(X, \beta^{\prime}\right) \times_{X} \bar{M}_{0, S^{\prime \prime} \cup\left\{x^{\prime \prime}\right\}}\left(X, \beta^{\prime \prime}\right) \longrightarrow \bar{M}_{0, S}(X, \beta)
$$

consisting of gluing together the two maps at $x^{\prime}$ and $x^{\prime \prime}$. We will assume that $S^{\prime}$ and $S^{\prime \prime}$ are nonempty and that $\beta^{\prime}$ and $\beta^{\prime \prime}$ are non-zero. Then $\rho_{D}$ is birational onto $D$. For short, let $\bar{M}^{\prime}$ and $\bar{M}^{\prime \prime}$ denote the factors of the fibred product above, and let

$$
\jmath_{D}: \bar{M}^{\prime} \times_{X} \bar{M}^{\prime \prime} \longrightarrow \bar{M}^{\prime} \times \bar{M}^{\prime \prime}
$$

denote the inclusion in the cartesian product.

Now there is the following formula for restricting a modified psi class to the boundary (assuming $\left.p_{i} \in S^{\prime}\right)$ :

$$
\rho_{D}^{*} \overline{\boldsymbol{\psi}}_{i}=\jmath_{D}^{*}\left(\overline{\boldsymbol{\psi}}_{i}+\boldsymbol{\delta}_{i x^{\prime}}\right) \text {. }
$$

Given generic even elements $\mathbf{x}, \mathbf{y} \in H^{*}(X, \mathbb{Q})$, we want to compute the integral of the class $\bar{\tau}_{0}(\mathbf{x})^{a} \bar{\tau}_{1}(\mathbf{y})^{b}$ over $D$. This depends on how the $a+b$ marks are distributed on the two twigs. 


\section{TANGENCY QUANTUM COHOMOLOGY}

Among the $a$ copies of $\bar{\tau}_{0}(\mathbf{x})$, assume $a^{\prime}$ of them belong to the $S^{\prime}$-twig and the remaining $a^{\prime \prime}$ classes fall on the $S^{\prime \prime}$-twig - similarly assume $b^{\prime}$ of the $b$ copies of $\bar{\tau}_{1}(\mathbf{y})$ sit on the $S^{\prime}$-twig and the remaining $b^{\prime \prime}$ on $S^{\prime \prime}$.

LEmma 1 (Splitting lemma for first enumerative descendants). In the situation just described we have

$$
\begin{aligned}
\left\langle D \cdot \bar{\tau}_{0}(\mathbf{x})^{a} \bar{\tau}_{1}(\mathbf{y})^{b}\right\rangle_{\beta}= & \sum_{p, q} \sum_{s^{\prime}, s^{\prime \prime}}\left(\begin{array}{c}
b^{\prime} \\
s^{\prime}
\end{array}\right)\left(\begin{array}{l}
b^{\prime \prime} \\
s^{\prime \prime}
\end{array}\right)\left\langle\bar{\tau}_{0}(\mathbf{x})^{a^{\prime}} \bar{\tau}_{1}(\mathbf{y})^{b^{\prime}-s^{\prime}} \bar{\tau}_{0}\left(\mathbf{y}^{s^{\prime}} \cup T_{p}\right)\right\rangle_{\beta^{\prime}} \\
& \times g^{p q}\left\langle\bar{\tau}_{0}\left(T_{q} \cup \mathbf{y}^{s^{\prime \prime}}\right) \bar{\tau}_{0}(\mathbf{x})^{a^{\prime \prime}} \bar{\tau}_{1}(\mathbf{y})^{b^{\prime \prime}-s^{\prime \prime}}\right\rangle_{\beta^{\prime \prime}}
\end{aligned}
$$

The outer sum is over the splitting indices $p$ and $q$ running from 0 to $r$, and the inner sum is over all non-negative integers $s^{\prime}$ and $s^{\prime \prime}$ : the symbol $\bar{\tau}_{1}(\mathbf{y})^{b^{\prime}-s^{\prime}}$ makes sense only for $s^{\prime} \leqslant b^{\prime}$, but since there is a binomial coefficient $\left(\begin{array}{c}b^{\prime} \\ s^{\prime}\end{array}\right)$ in front of it, which is zero unless $s^{\prime} \leqslant b^{\prime}$, we allow any $s^{\prime}$. Similarly for $s^{\prime \prime}$.

Proof. There are four ingredients in the proof of this formula: the first is of course the splitting axiom of Gromov-Witten theory (cf. Kontsevich and Manin [KM94]), which accounts for the overall shape of the formula. Second, formula (5) tells how each factor $\bar{\tau}_{1}(\mathbf{y})$ (say realised at mark $p_{i}$ as $\overline{\boldsymbol{\psi}}_{i} \cup \nu_{i}^{*}(\mathbf{y})$ ) restricts to give $\left(\overline{\boldsymbol{\psi}}_{i}+\boldsymbol{\delta}_{i x}\right) \cup \nu_{i}^{*}(\mathbf{y})$ on the twig containing $p_{i}$ - here $x$ denotes the gluing mark of that twig. Now (on each twig) expand the product of all these restrictions into a sum over $s$ (which accounts for the binomial coefficients). Third, apply formula (4) to write $\boldsymbol{\delta}_{i x} \cup \nu_{i}^{*}(\mathbf{y})=\boldsymbol{\delta}_{i x} \cup \nu_{x}^{*}(\mathbf{y})$, with the effect of accumulating evaluation classes on the gluing mark. Finally, by Equation (3) we can push down each term involving a diagonal class $\boldsymbol{\delta}_{i x}$ along the forgetful morphism forgetting $p_{i}$; the effect is simply deleting $\boldsymbol{\delta}_{i x}$.

\subsection{The tangency quantum potential}

The tangency quantum potential $\Gamma$ is now defined as the generating function for the first enumerative descendants. Let $\mathbf{x}=\sum x_{i} T_{i}$ and $\mathbf{y}=\sum y_{i} T_{i}$ be generic even elements of $H^{*}(X, \mathbb{Q})$, and set

$$
\begin{aligned}
\Gamma(\mathbf{x}, \mathbf{y}) & :=\sum_{\beta>0} q^{\beta}\left\langle\exp \left(\bar{\tau}_{0}(\mathbf{x})+\bar{\tau}_{1}(\mathbf{y})\right)\right\rangle_{\beta} \\
& =\sum_{\beta>0} q^{\beta} \sum_{a, b}\left\langle\frac{\bar{\tau}_{0}(\mathbf{x})^{a}}{a !} \frac{\bar{\tau}_{1}(\mathbf{y})^{b}}{b !}\right\rangle_{\beta} .
\end{aligned}
$$

The inner sum is over all non-negative integers $a$ and $b$ - this is meaningful since there is no $\beta=0$ term in the outer sum. (The degree-zero case is considered and included below, in $\S \S 4.3$ and 4.4.) The coefficients $q^{\beta}$ are necessary only to ensure formal convergence; they belong to the Novikov ring $\Lambda$ (as defined in Getzler [Get97]), which will be our coefficient ring when treating the tangency quantum potential.

For convenience we identify $\mathbf{x}$ and $\mathbf{y}$ with their coordinate vectors $\mathbf{x}=\left(x_{0}, \ldots, x_{r}\right)$ and $\mathbf{y}=$ $\left(y_{0}, \ldots, y_{r}\right)$ with respect to the basis $T_{0}, \ldots, T_{r}$. The potential thus belongs to the power series ring $\Lambda[[\mathbf{x}, \mathbf{y}]]=\Lambda\left[\left[x_{0}, \ldots, x_{r}, y_{0}, \ldots, y_{r}\right]\right]$, and expands to

$$
\Gamma(\mathbf{x}, \mathbf{y})=\sum_{\beta>0} q^{\beta} \sum_{\mathbf{a}, \mathbf{b}} \frac{\mathbf{y}^{\mathbf{b}}}{\mathbf{b} !} \frac{\mathbf{x}^{\mathbf{a}}}{\mathbf{a} !}\left\langle\overline{\boldsymbol{\tau}}_{0}^{\mathbf{a}} \overline{\boldsymbol{\tau}}_{1}^{\mathbf{b}}\right\rangle_{\beta} .
$$

Here the inner sum is over all pairs of vectors $\mathbf{a}=\left(a_{0}, \ldots, a_{r}\right)$ and $\mathbf{b}=\left(b_{0}, \ldots, b_{r}\right)$ of non-negative integers, and we employ multi-index notation, e.g. $\mathbf{a} !=a_{0} ! \cdots a_{r} !$. For the formal variables $\mathbf{x}$ and $\mathbf{y}$, the multi-index notation is reversed to preserve the signs arising from odd variables, e.g. $\mathbf{x}^{\mathbf{a}}=$ $x_{r}^{a_{r}} \cdots x_{0}^{a_{0}}$. 


\section{J. KOCK}

The variables $\mathbf{x}$ are the usual formal variables from quantum cohomology, so when $\mathbf{y}$ is set to zero, $\Gamma$ reduces to the usual (quantum part of the) genus-zero Gromov-Witten potential.

The viewpoint of Equation (7) is advantageous for the sake of extracting the invariants, as well as for checking the validity of certain formal operations on the potential. This task is safely left to the reader and henceforth only the more compact notation of Equation (6) is used.

\section{Deformation of the Poincaré metric}

While the usual quantum potential is based on the Poincaré metric constants $g_{i j}=\int_{X} T_{i} \cup T_{j}$, the tangency quantum potential relates more naturally to a deformation of them, a certain 'metric' with values in $\mathbb{Q}[[\mathbf{y}]]=\mathbb{Q}\left[\left[y_{0}, \ldots, y_{r}\right]\right]$. The basic properties of this deformed metric amount to formal manipulations with series and their derivatives. Although the arguments are rather straightforward, they are given in some detail, since the main result of this paper relies on the formalism.

\subsection{The classical product in the Poincaré metric}

The two important structures on $H=H^{*}(X, \mathbb{Q})$ are the intersection product $\cup$, and the trace map $\int_{X}: H \rightarrow \mathbb{Q}$ (integration over the fundamental class of $X$ ). Since this map is going to appear as the special fibre of a family of trace maps, we will henceforth denote it $\int_{0}: H \rightarrow \mathbb{Q}$; the generic fibre will then be denoted $\int_{\mathbf{y}}$, cf. $\S 2.4$. Set $g_{i j}=\int_{\mathbf{0}} T_{i} \cup T_{j}$ and $g_{i j k}=\int_{\mathbf{0}} T_{i} \cup T_{j} \cup T_{k}$. Let $\left(g^{i j}\right)$ be the inverse matrix to $\left(g_{i j}\right)$. It is used to raise indices as needed; in particular, with $g_{i j}^{k}=\sum_{e} g_{i j e} g^{e k}$, we have the multiplication formula

$$
T_{i} \cup T_{j}=\sum_{k} g_{i j}^{k} T_{k}
$$

\subsection{Intersection polynomials of $X$}

For a generic even element $\mathbf{y} \in H$ (identified with its coordinates $\mathbf{y}=\left(y_{0}, \ldots, y_{r}\right)$ with respect to $T_{0}, \ldots, T_{r}$ as in $\left.\S 1.5\right)$, let $\phi(\mathbf{y}) \in \mathbb{Q}[[\mathbf{y}]]$ be the generating function for the integrals on $X$,

$$
\begin{aligned}
\phi(\mathbf{y}):=\int_{\mathbf{0}} \exp (\mathbf{y}) & =\sum_{n \geqslant 0} \frac{1}{n !} \int_{\mathbf{0}} \mathbf{y}^{n} \\
& =\sum_{\mathbf{s}} \frac{y_{r}^{s_{r}}}{s_{r} !} \cdots \frac{y_{0}^{s_{0}}}{s_{0} !} \int_{\mathbf{0}} T_{0}^{s_{0}} \cdots T_{r}^{s_{r}} .
\end{aligned}
$$

Note that this series is $\exp \left(y_{0}\right)$ times a polynomial in $y_{1}, \ldots, y_{r}$. The heavy explicit notation of the last expression is avoided in the following - the reader can revert to this notation if needed.

Let subscripts denote partial differentiation as follows:

$$
\phi_{i}:=\frac{\partial}{\partial y_{i}} \phi=\int_{\mathbf{0}} \exp (\mathbf{y}) \cup T_{i}, \quad \phi_{i j}:=\frac{\partial^{2}}{\partial y_{i} \partial y_{j}} \phi=\int_{\mathbf{0}} \exp (\mathbf{y}) \cup T_{i} \cup T_{j} .
$$

(Note that $\exp (\mathbf{y})$ is even, so we could also write $\phi_{i}=\int_{\mathbf{0}} T_{i} \cup \exp (\mathbf{y})$, but among the indices the order is significant.)

Use the matrix $\left(g^{e f}\right)$ to raise indices, putting

$$
\phi_{j}^{i}:=\sum_{e} g^{i e} \phi_{e j}, \quad \phi_{i}{ }^{j}:=\sum_{f} \phi_{i f} g^{f j}, \quad \phi^{i j}:=\sum_{e, f} g^{i e} \phi_{e f} g^{f j} .
$$

The entities $\phi_{j}^{i}(\mathbf{y})$ (respectively $\phi_{i}^{j}(\mathbf{y})$ ) are important because they are the tensor elements of 
'multiplication by $\exp (\mathbf{y})$ ':

$$
\begin{aligned}
\exp (\mathbf{y}) \cup T_{p} & =\sum_{e, f} T_{e} g^{e f} \int_{\mathbf{0}} T_{f} \cup \exp (\mathbf{y}) \cup T_{p} \\
& =\sum_{e} T_{e} \phi^{e}{ }_{p}(\mathbf{y})
\end{aligned}
$$

(respectively $\left.T_{q} \cup \exp (\mathbf{y})=\sum_{f} \phi_{q}^{f}(\mathbf{y}) T_{f}\right)$. (These are identities in $\left.H[[\mathbf{y}]].\right)$

We will also need symbols with three indices. Specifically, starting from the third derivatives

$$
\phi_{i j k}:=\frac{\partial^{3}}{\partial y_{i} \partial y_{j} \partial y_{k}} \phi,
$$

the outer indices can be raised as in Equation (9) - for example

$$
\phi_{j}^{i k}:=\sum_{e, f} g^{i e} \phi_{e j f} g^{f k}
$$

Observe that for all $\phi$-symbols, upon setting $\mathbf{y}=\mathbf{0}$ we recover the corresponding $g$-symbol. For example, we have

$$
\phi_{j k}^{i}(\mathbf{0})=g_{j k}^{i} .
$$

The following formula is the basic instance of an important principle. It can be used to show that many other index-raising rules (contractions) hold as expected.

Lemma 2 (Sum formula). Let $\mathbf{y}^{\prime}$ and $\mathbf{y}^{\prime \prime}$ be generic even elements of $H$. Then

$$
\phi\left(\mathbf{y}^{\prime}+\mathbf{y}^{\prime \prime}\right)=\sum_{e, f} \phi_{e}\left(\mathbf{y}^{\prime}\right) g^{e f} \phi_{f}\left(\mathbf{y}^{\prime \prime}\right) .
$$

Proof. Consider the product manifold with its projections

$$
X \stackrel{p^{\prime}}{\longleftarrow} X \times X \stackrel{p^{\prime \prime}}{\longrightarrow} X .
$$

For each $i=0, \ldots, r$, put $T_{i}^{\prime}:=p^{*} T_{i}$ and $T_{i}^{\prime \prime}:=p^{\prime \prime *} T_{i}$; so that $T_{0}^{\prime}, \ldots, T_{r}^{\prime}, T_{0}^{\prime \prime}, \ldots, T_{r}^{\prime \prime}$ is a basis for $X \times X$. Now write down the exponential series for $X \times X$

$$
\exp \left(\mathbf{y}^{\prime}+\mathbf{y}^{\prime \prime}\right)=\exp \left(\sum y_{i}^{\prime} T_{i}^{\prime}+\sum y_{i}^{\prime \prime} T_{i}^{\prime \prime}\right)
$$

and integrate over the diagonal $\Delta$. Computing this integral using the isomorphism $X \simeq \Delta$ yields the left-hand side of the desired equation. On the other hand, computing the integral using the Künneth decomposition $[\Delta]=\sum_{e, f} T_{e}^{\prime} g^{e f} T_{f}^{\prime \prime}$ we get

$$
\begin{aligned}
\int_{\Delta} \exp \left(\mathbf{y}^{\prime}+\mathbf{y}^{\prime \prime}\right) & =\int_{X \times X} \exp \left(\mathbf{y}^{\prime}\right) \cup[\Delta] \cup \exp \left(\mathbf{y}^{\prime \prime}\right) \\
& =\sum_{e, f}\left(\int_{X} \exp \left(\mathbf{y}^{\prime}\right) \cup T_{e}\right) g^{e f}\left(\int_{X} T_{f} \cup \exp \left(\mathbf{y}^{\prime \prime}\right)\right)
\end{aligned}
$$

via the projection formula, arriving at the right-hand side.

\subsection{Variations on the sum formula}

The same principle readily yields many other useful formulae. For instance, applying the argument to the series $\exp \left(\mathbf{y}^{\prime}+\mathbf{y}^{\prime \prime}\right) \cup T_{i}^{\prime} \cup T_{j}^{\prime \prime}$ gives the formula

$$
\phi_{i j}\left(\mathbf{y}^{\prime}+\mathbf{y}^{\prime \prime}\right)=\sum_{e, f} \phi_{i e}\left(\mathbf{y}^{\prime}\right) g^{e f} \phi_{f j}\left(\mathbf{y}^{\prime \prime}\right),
$$




\section{J. KOCK}

and if instead we start with $\exp \left(\mathbf{y}^{\prime}+\mathbf{y}^{\prime \prime}\right) \cup T_{i}^{\prime} \cup T_{j}^{\prime} \cup T_{k}^{\prime \prime}$ we arrive at

$$
\phi_{i j k}\left(\mathbf{y}^{\prime}+\mathbf{y}^{\prime \prime}\right)=\sum_{e, f} \phi_{i j e}\left(\mathbf{y}^{\prime}\right) g^{e f} \phi_{f k}\left(\mathbf{y}^{\prime \prime}\right)
$$

These formulae can then have their outer indices raised as in Equations (9) and (11), yielding

$$
\begin{aligned}
\phi^{i j}\left(\mathbf{y}^{\prime}+\mathbf{y}^{\prime \prime}\right) & =\sum_{e, f} \phi_{e}^{i}\left(\mathbf{y}^{\prime}\right) g^{e f} \phi_{f}^{j}\left(\mathbf{y}^{\prime \prime}\right) \\
\phi_{j}^{i k}\left(\mathbf{y}^{\prime}+\mathbf{y}^{\prime \prime}\right) & =\sum_{e, f} \phi_{j e}^{i}\left(\mathbf{y}^{\prime}\right) g^{e f} \phi_{f}^{k}\left(\mathbf{y}^{\prime \prime}\right) .
\end{aligned}
$$

Finally, useful formulae drop out by specialising $\mathbf{y}^{\prime}$ or $\mathbf{y}^{\prime \prime}$. For example, setting $\mathbf{y}^{\prime}=\mathbf{0}$ and $\mathbf{y}^{\prime \prime}=\mathbf{y}$ in Equation (16) we get

$$
\phi_{j}^{i}{ }^{k}=\phi_{j}^{i}{ }^{k}(\mathbf{0}+\mathbf{y})=\sum_{e, f} \phi_{j e}^{i}(\mathbf{0}) g^{e f} \phi_{f}^{k}(\mathbf{y})=\sum_{e} g_{j e}^{i} \phi^{e k}
$$

via Equations (12) and (9). Similarly, $\phi^{i}{ }_{j}^{k}=\sum_{f} \phi^{i f} g_{f j}^{k}$.

\subsection{The deformed metric}

Instead of using the integral $\int_{\mathbf{0}}: H \rightarrow \mathbb{Q}$, the new metric is based on the linear map $\int_{\mathbf{y}}: H \rightarrow \mathbb{Q}[[\mathbf{y}]]$ defined as

$$
\int_{\mathbf{y}} \mathbf{z}:=\int_{\mathbf{0}} \exp (-2 \mathbf{y}) \cup \mathbf{z}
$$

It is thought of as a deformation of $\int_{\mathbf{0}}$ since we recover this map upon setting $\mathbf{y}=\mathbf{0}$. Now define the new metric $\left(\gamma_{i j}\right)$ by

$$
\gamma_{i j}:=\gamma_{i j}(\mathbf{y}):=\int_{\mathbf{y}} T_{i} \cup T_{j}=\phi_{i j}(-2 \mathbf{y})
$$

and adopt the obvious notation $\gamma_{i j k}=\int_{\mathbf{y}} T_{i} \cup T_{j} \cup T_{k}=\phi_{i j k}(-2 \mathbf{y})$. Let $\left(\gamma^{i j}\right)$ denote the inverse matrix to $\left(\gamma_{i j}\right)$. Then it follows readily from the sum formula (and Equation (15)) that

$$
\gamma^{i j}=\phi^{i j}(2 \mathbf{y})=\sum_{e, f} \phi^{i}{ }_{e}^{e f} \phi_{f}^{j}
$$

This formula is the most important expression for the deformed metric, since it is in this form it will appear in the proof of Theorem 1. (Note that the indices on $\gamma$ do not denote derivatives, and that the upper indices are not just raised by $\left(g^{e f}\right)$. Specifically, $\gamma^{i j}$ is not equal to $\sum_{e, f} g^{i e} \gamma_{e f} g^{f j}$.)

The sum formula also yields $\sum_{e} \gamma_{i j e} \gamma^{e f}=\sum_{e} \phi_{i j e}(-2 \mathbf{y}) \phi^{e f}(2 \mathbf{y})=\phi_{i j}{ }^{f}(\mathbf{0})=g_{i j}^{f}$, thus the intersection product can be written

$$
T_{i} \cup T_{j}=\sum_{e, f} \gamma_{i j e} \gamma^{e f} T_{f} .
$$

\subsection{Example}

For $\mathbb{P}^{2}\left(\right.$ with $h:=c_{1}(\mathcal{O}(1))$ and basis $\left.T_{i}:=h^{i}\right)$, we get

$$
\left(\gamma^{i j}\right)=\exp \left(2 y_{0}\right)\left(\begin{array}{ccc}
0 & 0 & 1 \\
0 & 1 & 2 y_{1} \\
1 & 2 y_{1} & 2 y_{1}^{2}+2 y_{2}
\end{array}\right) .
$$

This matrix (with $y_{0}$ and $y_{2}$ set to zero) was first written down in the pioneering article of di Francesco and Itzykson [dFI95]. 


\section{WDVV equations and the tangency quantum product}

\subsection{Notation}

Let the lower indices on $\Gamma$ denote partial derivatives with respect to the $x$-variables, for example

$$
\Gamma_{i j}:=\Gamma_{x_{i} x_{j}}:=\frac{\partial^{2}}{\partial x_{i} \partial x_{j}} \Gamma=\sum_{\beta>0} q^{\beta}\left\langle\exp \left(\bar{\tau}_{0}(\mathbf{x})+\bar{\tau}_{1}(\mathbf{y})\right) \cdot \bar{\tau}_{0}\left(T_{i}\right) \bar{\tau}_{0}\left(T_{j}\right)\right\rangle_{\beta},
$$

and set

$$
\Gamma_{(i j)}=\Gamma_{\left(x_{i} x_{j}\right)}:=\sum_{k=0}^{r} \Gamma_{x_{k}} g_{i j}^{k},
$$

as the 'directional derivative with respect to the product $T_{i} \cup T_{j}=\sum g_{i j}^{k} T_{k}$ '.

THEOREM 1. The following form of the WDVV equations holds for the tangency quantum potential.

$$
\Gamma_{(i j) k \ell}+\Gamma_{i j(k \ell)}+\sum_{e, f} \Gamma_{i j e} \gamma^{e f} \Gamma_{f k \ell}= \pm\left(\Gamma_{(j k) i \ell}+\Gamma_{j k(i \ell)}+\sum_{e, f} \Gamma_{j k e} \gamma^{e f} \Gamma_{f i \ell}\right),
$$

where ' \pm ' denotes the sign of $(-1)^{\operatorname{deg} T_{i}\left(\operatorname{deg} T_{j}+\operatorname{deg} T_{k}\right)}$.

Proof. The proof follows the line of arguments of the proof of the WDVV equations for the usual Gromov-Witten potential, cf. Kontsevich and Manin [KM94]. The novelty is the splitting lemma for enumerative descendants and the appearance of the deformed metric.

For fixed degree $\beta>0$ and integers $a, b \geqslant 0$, consider the moduli stack $\bar{M}_{0,\left\{p_{1}, p_{2}, p_{3}, p_{4}\right\} \cup S}(X, \beta)$ where $S$ is a marking set of cardinality $a+b$. Consider the product

$$
\bar{\tau}_{0}\left(T_{i}\right) \bar{\tau}_{0}\left(T_{j}\right) \bar{\tau}_{0}\left(T_{k}\right) \bar{\tau}_{0}\left(T_{\ell}\right) \frac{\bar{\tau}_{0}(\mathbf{x})^{a}}{a !} \frac{\bar{\tau}_{1}(\mathbf{y})^{b}}{b !}
$$

where the first four classes correspond to the marks $p_{1}, p_{2}, p_{3}, p_{4}$. Now integrate the product over each side of the fundamental equivalence

$$
\left(p_{1}, p_{2} \mid p_{3}, p_{4}\right)=\left(p_{2}, p_{3} \mid p_{1}, p_{4}\right)
$$

where $\left(p_{1}, p_{2} \mid p_{3}, p_{4}\right)$ denotes the sum of all boundary divisors having $p_{1}$ and $p_{2}$ on one twig and $p_{3}$ and $p_{4}$ on the other. Summing up these equations over all $a, b \geqslant 0$ and over all $\beta>0$ (as in the definition of $\Gamma$ ) we will get the desired equation. Let us treat the left-hand side. On the right-hand side of the equation the arguments are the same; only it is necessary initially to permute the four special factors, which accounts for the sign (as in [KM94]).

On the left-hand side of the equation, let us first consider the contribution from the trivial degree partitions, say $\beta^{\prime}=0$. Then the only possible distribution of the marks giving a contribution is when all the spare marks fall on the right-hand twig, which leaves us with the single boundary divisor $D_{12}$. Now according to $\S 1.3$, the effect of multiplication with this divisor is to merge the two classes $\bar{\tau}_{0}\left(T_{i}\right)$ and $\bar{\tau}_{0}\left(T_{j}\right)$ and so in the end we get

$$
\left\langle\bar{\tau}_{0}\left(T_{i} \cup T_{j}\right) \bar{\tau}_{0}\left(T_{k}\right) \bar{\tau}_{0}\left(T_{\ell}\right) \frac{\bar{\tau}_{0}(\mathbf{x})^{a}}{a !} \frac{\bar{\tau}_{1}(\mathbf{y})^{b}}{b !}\right\rangle_{\beta} .
$$

Similarly, the case $\beta^{\prime \prime}=0$ gives

$$
\left\langle\bar{\tau}_{0}\left(T_{i}\right) \bar{\tau}_{0}\left(T_{j}\right) \bar{\tau}_{0}\left(T_{k} \cup T_{\ell}\right) \frac{\bar{\tau}_{0}(\mathbf{x})^{a}}{a !} \frac{\bar{\tau}_{1}(\mathbf{y})^{b}}{b !}\right\rangle_{\beta} .
$$

Summing over $\beta>0$ and $a, b \geqslant 0$ gives exactly the two linear terms on the left-hand side of the promised equation, cf. Equation (19). 


\section{J. KOCK}

Now for those boundary divisors in the linear equivalence corresponding to strictly positive degree partitions. To each irreducible component on the left-hand side of Equation (20), we apply the Splitting Lemma 1, getting all together

$$
\begin{aligned}
\sum \sum_{p, q}\left\langle\frac{\bar{\tau}_{0}(\mathbf{x})^{a^{\prime}}}{a^{\prime} !} \frac{\bar{\tau}_{1}(\mathbf{y})^{b^{\prime}}}{b^{\prime} !} \bar{\tau}_{0}\left(T_{i}\right) \bar{\tau}_{0}\left(T_{j}\right) \bar{\tau}_{0}\left(\frac{\mathbf{y}^{c^{\prime}}}{c^{\prime} !} \cup T_{p}\right)\right\rangle_{\beta^{\prime}} \\
\\
\times g^{p q}\left\langle\bar{\tau}_{0}\left(T_{q} \cup \frac{\mathbf{y}^{c^{\prime \prime}}}{c^{\prime \prime} !}\right) \bar{\tau}_{0}\left(T_{k}\right) \bar{\tau}_{0}\left(T_{\ell}\right) \frac{\bar{\tau}_{0}(\mathbf{x})^{a^{\prime \prime}}}{a^{\prime \prime} !} \frac{\bar{\tau}_{1}(\mathbf{y})^{b^{\prime \prime}}}{b^{\prime \prime} !}\right\rangle_{\beta^{\prime \prime}} .
\end{aligned}
$$

where the big outer sum is over all $a^{\prime}+a^{\prime \prime}=a$ and all $b^{\prime}+c^{\prime}+b^{\prime \prime}+c^{\prime \prime}=b$. (The $b^{\prime}$ corresponds to what was called $b^{\prime}-s^{\prime}$ in the Splitting Lemma 1.) Now sum over all $a$ and $b$ getting

$$
\begin{aligned}
\sum_{p, q}\left\langle\exp \left(\bar{\tau}_{0}(\mathbf{x})+\bar{\tau}_{1}(\mathbf{y})\right) \cdot \bar{\tau}_{0}\left(T_{i}\right) \bar{\tau}_{0}\left(T_{j}\right) \bar{\tau}_{0}\left(\exp (\mathbf{y}) \cup T_{p}\right)\right\rangle_{\beta^{\prime}} & \\
& \times g^{p q}\left\langle\bar{\tau}_{0}\left(T_{q} \cup \exp (\mathbf{y})\right) \bar{\tau}_{0}\left(T_{k}\right) \bar{\tau}_{0}\left(T_{\ell}\right) \cdot \exp \left(\bar{\tau}_{0}(\mathbf{x})+\bar{\tau}_{1}(\mathbf{y})\right)\right\rangle_{\beta^{\prime \prime}}
\end{aligned}
$$

Next, use Equation (10) to get rid of $\exp (\mathbf{y})$, and sum over all $\beta>0$ as in the definition of $\Gamma$, arriving at

$$
\sum_{\substack{p, q \\ e, f}} \Gamma_{i j e} \phi^{e}{ }_{p} g^{p q} \phi_{q}{ }^{f} \Gamma_{f k \ell} .
$$

By Equation (18), this is just the quadratic term of the left-hand side of the desired equation.

\subsection{Enumerative geometry}

According to [GKP02, Lemma 3.2.3], when $X$ is a homogeneous variety, the tangency quantum potential is related to the rational characteristic number potential by a linear change of coordinates of the form $x \mapsto x+\rho y, y \mapsto \sigma y$. Since the derivatives appearing in the above WDVV equation are only with respect to $x$-variables, it follows that the characteristic number potential satisfies essentially the same equation. For $X=\mathbb{P}^{r}$, the equation is exactly the same. For more complicated varieties (like $\mathbb{P}^{1} \times \mathbb{P}^{1}$ ) the definition of characteristic numbers involves the choice of which hypersurfaces are used to impose tangency conditions. Depending on this choice, the substitution $y \mapsto \sigma y$ which must be performed in $\gamma$ can be non-trivial, so the deformed metric appearing in WDVV for the characteristic number potential is slightly different in that case. These coordinate changes are carefully explained in [GKP02].

\subsection{Example}

In the special case $X=\mathbb{P}^{2}$, it turns out the only non-trivial relation in Theorem 1 is the one with $i=j=1$ and $k=\ell=2$. Since $\Gamma_{0}=0$ by the string equation (cf. [GKP02]), the relation reads

$$
\Gamma_{222}=\exp \left(2 y_{0}\right)\left(\Gamma_{112}^{2}-\Gamma_{111} \Gamma_{122}+2 y_{1}\left(\Gamma_{122} \Gamma_{112}-\Gamma_{111} \Gamma_{222}\right)+\left(2 y_{1}^{2}+2 y_{2}\right)\left(\Gamma_{122}^{2}-\Gamma_{112} \Gamma_{222}\right)\right) .
$$

This equation (for the characteristic number potential) was first found by Ernström and Kennedy [EK99] (cf. Remark 1 below), while the special case of $y_{0}=y_{2}=0$ goes back to di Francesco and Itzykson [dFI95]. By also setting $y_{1}=0$ we are back to the celebrated formula of Kontsevich [KM94].

\subsection{Topological recursion relation}

The deformed WDVV equations given in $\S 3.3$ alone are not sufficient to determine all the first enumerative descendants (or the characteristic numbers) from the primary Gromov-Witten invariants. But topological recursion is also available for the enumerative descendants, cf. [GKP02]. 


\section{TANGENCY QUANTUM COHOMOLOGY}

In the present set-up, that topological recursion relation takes the following pleasant form:

$$
\Gamma_{y_{i} x_{j} x_{k}}=\Gamma_{x_{i}\left(x_{j} x_{k}\right)}-\Gamma_{\left(x_{i} x_{j}\right) x_{k}}-\Gamma_{\left(x_{i} x_{k}\right) x_{j}}+\sum_{e, f} \Gamma_{x_{i} x_{e}} \gamma^{e f} \Gamma_{x_{f} x_{j} x_{k}}
$$

The shape of this equation stems from the boundary expression of the modified psi class, $\overline{\boldsymbol{\psi}}_{i}=$ $\left(p_{i} \mid p_{j}, p_{k}\right)-\boldsymbol{\xi}_{i}$, where $\boldsymbol{\xi}_{i}$ is the sum of all boundary divisors such that $p_{i}$ is on a contracting twig. The deformed metric enters in the quadratic terms for the same reason as in the proof in $\S 3.1$.

\subsection{The tangency quantum product}

The tangency quantum product '*' is the $\Lambda[[\mathbf{x}, \mathbf{y}]]$-bilinear product on $H^{*}(X, \mathbb{Q}) \otimes_{\mathbb{Q}} \Lambda[[\mathbf{x}, \mathbf{y}]]$ defined by the rule

$$
T_{i} * T_{j}:=T_{i} \cup T_{j}+\sum_{e, f} \Gamma_{i j e} \gamma^{e f} T_{f} .
$$

Clearly this product is supercommutative. Furthermore, since the 'string equation' for $\Gamma$ is simply $\Gamma_{0}=0$, it follows that $T_{0}$ is the 1-element for $*$. Note also that the product specialises to the usual quantum product upon setting the formal variables $\mathbf{y}$ to zero.

THEOREM 2. The tangency quantum product is associative.

Proof. This is a straightforward consequence of Theorem 1; it amounts to checking the associativity relations on the generators, using the definition of the product. The only subtle point in the verification is the identity

$$
\sum_{e, f} \Gamma_{i j e} \gamma^{e f}\left(T_{f} \cup T_{k}\right)=\sum_{\ell, m} \Gamma_{i j(k \ell)} \gamma^{\ell m} T_{m}
$$

which follows from the properties of the structure constants $g_{i j}^{k}$. First apply Equation (8) to write $\left(T_{f} \cup T_{k}\right)=\sum_{m} g_{f k}^{m} T_{m}$. Then use Equation (17) to write $\sum_{f} \gamma^{e f} g_{f k}^{m}=\sum_{\ell} g_{k \ell}^{e} \gamma^{\ell m}$; and finally use (the derivative of) Equation (19) to write $\sum_{e} \Gamma_{i j e} g_{k \ell}^{e}=\Gamma_{i j(k \ell)}$.

Remark 1 . In the special case $X=\mathbb{P}^{2}$, this product was previously constructed via ad hoc methods by Ernström and Kennedy [EK99], who also gave a tour de force proof of its associativity. Their construction relies on the space of stable lifts, and seems to be peculiar to the projective plane.

\section{Integrability}

\subsection{The classical potential}

The classical potential for $X$ is the generating function for the triple top products

$$
\int_{\mathbf{0}} \frac{\mathbf{x}^{3}}{3 !}=\sum_{i, j, k} \frac{x_{k} x_{j} x_{i}}{6} \int_{\mathbf{0}} T_{i} \cup T_{j} \cup T_{k} .
$$

By construction, its third derivatives are just $g_{i j k}$, the structure constants for the cup multiplication (in the Poincaré metric). In usual quantum cohomology, this potential is reinterpreted as the $\beta=0$ part of the Gromov-Witten potential: a quantum correction (the $\beta>0$ part) is added to the classical potential, in such a way that the third derivatives of this sum are the structure constants of a new associative product - the quantum product. 


\section{J. KOCK}

\section{2 'The tangency classical potential'}

The tangency quantum product relates to $\int_{\mathbf{y}}$ exactly as the usual quantum product relates to $\int_{\mathbf{0}}$. So let us introduce a potential

$$
\Phi^{0}(\mathbf{x}, \mathbf{y}):=\int_{\mathbf{y}} \frac{\mathbf{x}^{3}}{3 !}=\sum_{i, j, k} \frac{x_{k} x_{j} x_{i}}{6} \int_{\mathbf{y}} T_{i} \cup T_{j} \cup T_{k},
$$

which to fit into the picture could be called the tangency classical potential, although it is neither classical, nor has anything particular to do with tangency. By construction, its third derivatives are $\gamma_{i j k}$, the structure constants of the cup multiplication, but this time in the deformed metric

$$
T_{i} \cup T_{j}=\sum_{e, f} \Phi_{i j e}^{0} \gamma^{e f} T_{f}
$$

\subsection{The tangency potential, including $\beta=0$}

Introducing the potential

$$
\Phi(\mathbf{x}, \mathbf{y}):=\Phi^{0}(\mathbf{x}, \mathbf{y})+\Gamma(\mathbf{x}, \mathbf{y}),
$$

whose third derivatives are $\Phi_{i j k}=\gamma_{i j k}+\Gamma_{i j k}$, the tangency quantum product can be written

$$
T_{i} * T_{j}=\sum_{e, f} \Phi_{i j e} \gamma^{e f} T_{f},
$$

and the WDVV equation of Theorem 1 then takes the usual form

$$
\sum_{e, f} \Phi_{i j e} \gamma^{e f} \Phi_{f k \ell}= \pm \sum_{e, f} \Phi_{j k e} \gamma^{e f} \Phi_{f i \ell}
$$

\subsection{Interpretation of the degree-zero term}

Writing down this potential $\Phi=\Phi^{0}+\Gamma$ calls for an interpretation of $\Phi^{0}$ in terms of some top products on the degree-zero spaces. Unfortunately there is no way of defining the modified psi class on $\bar{M}_{0, n}$, if we want it to satisfy the two rules:

i) it should be compatible with pull-back along forgetful morphisms; and

ii) it should satisfy the push-down formula $\pi_{*} \bar{\psi}=-2$, independent of the number of marks, to give the dilaton equation for modified psi classes (cf. [GKP02]).

Basically this is impossible because the one-pointed spaces do not exist in degree zero. The best one can do is to define the class on a fixed four-pointed space and then pull it back to the hierarchy lying over this space, but this definition depends on the choice of the three extra marks.

So take a moduli space $\bar{M}_{0, S \cup\left\{q_{1}, q_{2}, q_{3}\right\}}(X, 0)$ with three distinguished marks. For each of the other marks $p_{s} \in S$, define the modified psi class $\overline{\boldsymbol{\psi}}_{s}$ as the pull-back from $\bar{M}_{0,\left\{p_{s}, q_{1}, q_{2}, q_{3}\right\}} \simeq \mathbb{P}^{1}$ of the class of degree -2 . One easily checks that this is equivalent to defining $\overline{\boldsymbol{\psi}}_{s}:=\boldsymbol{\psi}_{s}-\boldsymbol{\xi}_{s}$, where $\boldsymbol{\xi}_{s}$ is the sum of all boundary divisors such that $p_{s}$ is on a (contracting) twig together with at most one of the distinguished marks. (This description is then compatible with the boundary description of the modified psi class in the $\beta>0$ case (cf. [GKP02]), since in that case there are no distinguished marks.)

In this setting, define the invariant

$$
\left\langle\frac{\bar{\tau}_{0}(\mathbf{x})^{a}}{a !} \frac{\bar{\tau}_{1}(\mathbf{y})^{b}}{b !} \bar{\tau}_{0}\left(T_{i}\right) \bar{\tau}_{0}\left(T_{j}\right) \bar{\tau}_{0}\left(T_{k}\right)\right\rangle_{0}
$$




\section{TANGENCY QUANTUM COHOMOLOGY}

in the obvious way, with the last three classes corresponding to the three distinguished marks. Identifying $\bar{M}_{0, S \cup\left\{q_{1}, q_{2}, q_{3}\right\}}(X, 0)$ with $\bar{M}_{0, S \cup\left\{q_{1}, q_{2}, q_{3}\right\}} \times X$, all the evaluation morphisms are just the projection $p$ to $X$, so the integrand has a factor $p^{*}\left(\left(\mathbf{x}^{a} / a !\right) \cup\left(\mathbf{y}^{b} / b !\right) \cup T_{i} \cup T_{j} \cup T_{k}\right)$. The remaining factors are modified psi classes from $\bar{M}_{0, S \cup\left\{q_{1}, q_{2}, q_{3}\right\}}$; since each of them is alone on its mark, we can push them down one by one, arriving at a factor of $(-2)^{b}$. The remaining integral $\int 1$ is zero for dimensional reasons, unless we have come down to just $\bar{M}_{0,\left\{q_{1}, q_{2}, q_{3}\right\}}$, which means $a=0$. We conclude

$$
\left\langle\frac{\bar{\tau}_{1}(\mathbf{y})^{b}}{b !} \bar{\tau}_{0}\left(T_{i}\right) \bar{\tau}_{0}\left(T_{j}\right) \bar{\tau}_{0}\left(T_{k}\right)\right\rangle_{0}=\int_{0} \frac{(-2 \mathbf{y})^{b}}{b !} \cup T_{i} \cup T_{j} \cup T_{k} .
$$

Summing over all $b$ (and $a$ ) we get

$$
\int_{\mathbf{0}} \exp (-2 \mathbf{y}) \cup T_{i} \cup T_{j} \cup T_{k}=\int_{\mathbf{y}} T_{i} \cup T_{j} \cup T_{k}=\gamma_{i j k}
$$

showing that at least the third derivatives of $\Phi^{0}$ have an interpretation as top products on degreezero moduli spaces and, after all, it is the third derivatives that really matter.

Once we know that the structure constants of the tangency quantum product are third derivatives of the single potential $\Phi$, we are in position to give an interpretation in terms of Frobenius manifolds.

\subsection{Formal Frobenius manifolds}

For convenience let us recall (from Manin [Man99], Ch. III) the definition of a formal Frobenius manifold over a base ring. Let $k$ be a supercommutative $\mathbb{Q}$-algebra. Let $H$ be a free $k$-module of finite rank, with generators $T_{0}, \ldots, T_{r}$, and let $g: H \otimes H \rightarrow k$ denote an even symmetric non-degenerate bilinear pairing. Let $K=k\left[\left[H^{t}\right]\right]$ be the completed symmetric algebra of the dual module $H^{t}$. In other words, if $\mathbf{x}=\sum x_{i} T_{i}$ is a generic even element of $H$ then $K=k\left[\left[x_{0}, \ldots, x_{r}\right]\right]$. Now the structure of a formal Frobenius manifold on $(H, g)$ over $k$ is given by an even potential $\Phi \in K$ (defined up to quadratic terms) satisfying WDVV. In other words, the multiplication $T_{i} * T_{j}:=$ $\sum_{e, f} \Phi_{i j e} g^{e f} T_{f}$ makes $H \otimes_{k} K$ into an associative supercommutative $K$-algebra.

With $H:=H^{*}(X, \Lambda)$, the results of this section readily imply the following.

Theorem 3. The cohomology $\Lambda[[\mathbf{y}]]$-module $H[[\mathbf{y}]]$ with bilinear non-degenerate pairing $\gamma: H[[\mathbf{y}]] \otimes$ $H[[\mathbf{y}]] \rightarrow \Lambda[[\mathbf{y}]]$, equipped with the tangency quantum potential $\Phi \in \Lambda[[\mathbf{x}, \mathbf{y}]]$ constitutes a formal Frobenius manifold over $\Lambda[[\mathbf{y}]]$, with $T_{0}$ as the identity.

In fact, this formal Frobenius manifold is a deformation over $\mathbb{Q}[[\mathbf{y}]]$ of the formal Frobenius manifold of usual quantum cohomology. While the underlying space is trivially deformed under this deformation, the metric and the potential vary non-trivially.

\section{ACKNOWLEDGEMEnts}

This work was part of my PhD thesis [Koc00], and I am indebted to my advisor Israel Vainsencher for his guidance. This particular part was carried out while I was visiting the California Institute of Technology; I am thankful to Caltech for its hospitality, and in particular to Rahul Pandharipande for encouraging my research, and for many stimulating conversations. I have also benefited from conversations with Marzia Polito and André Hirschowitz, and correspondence with Gary Kennedy and Ezra Getzler. 


\section{TANGENCY QUANTUM COHOMOLOGY}

\section{REFERENCES}

Beh97 K. Behrend, Gromov-Witten invariants in algebraic geometry, Invent. Math. 127 (1997), 601-617 (alg-geom/9601011).

BM96 K. Behrend and Yu. I. Manin, Stacks of stable maps and Gromov-Witten invariants, Duke. J. Math. 85 (1996), 1-60 (alg-geom/9506023).

dFI95 P. di Francesco and C. Itzykson, Quantum intersection rings, in The moduli space of curves, Progress in Mathematics, vol. 129, eds R. Dijkgraaf, C. Faber and G. van der Geer (Birkhäuser, Boston, MA, 1995), 81-148.

Dub96 B. Dubrovin, Geometry of $2 D$ topological field theories, in Integrable systems and quantum groups, Lecture Notes in Mathematics, vol. 1620 (Springer, Berlin, 1996), 120-348 (hep-th/9407018).

EK99 L. Ernström and G. Kennedy, Contact cohomology of the projective plane, Amer. J. Math. 121 (1999), 73-96 (alg-geom/9703013).

FP95 W. Fulton and R. Pandharipande, Notes on stable maps and quantum cohomology, in Algebraic geometry, Santa Cruz 1995, Proc. Symp. Pure. Math., vol. 62(II), eds J. Kollár, R. Lazarsfeld and D. Morrison (1996), 45-96 (alg-geom/9608011).

Get97 E. Getzler, Intersection theory on $\bar{M}_{1,4}$ and elliptic Gromov-Witten invariants, J. Amer. Math. Soc. 10 (1997), 973-998 (alg-geom/9612004).

GKP02 T. Graber, J. Kock, and R. Pandharipande, Descendant invariants and characteristic numbers, Amer. J. Math. 124 (2002), 611-647 (math.AG/0102017).

Koc00 J. Kock, Tangency quantum cohomology and enumerative geometry of rational curves, PhD thesis, Recife, Brazil (2000).

Koc03 J. Kock, Characteristic numbers of rational curves with cusp or prescribed triple contact. Math. Scand. 92 (2003), 223-245 (math.AG/0102082).

KM94 M. Kontsevich and Yu. I. Manin, Gromov-Witten classes, quantum cohomology, and enumerative geometry, Comm. Math. Phys. 164 (1994), 525-562 (hep-th/9402147).

LT98 J. Li and G. Tian, Virtual moduli cycles and Gromov-Witten invariants of algebraic varieties, J. Amer. Math. Soc. 11 (1998), 119-174 (alg-geom/9602007).

Man99 Yu. I. Manin, Frobenius manifolds, quantum cohomology, and moduli spaces, AMS Colloquium Publications (American Mathematical Society, Providence, RI, 1999).

Wit91 E. Witten, Two-dimensional gravity and intersection theory on moduli space, Surveys Diff. Geom. 1 (1991), 243-310.

Joachim Kock jojo@dmat.ufpe.br

Departamento de Matemática, Universidade Federal de Pernambuco, Cidade Universitária 50670-

901, Recife, PE, Brazil 